\title{
Health-related quality of life in thrombocytopenic patients with chronic hepatitis $C$ with or without cirrhosis in the ENABLE-1 and ENABLE-2 studies
}

Kelly M. Grotzinger ${ }^{1 *}$, Zobair M. Younossi ${ }^{2}$, Edoardo G. Giannini ${ }^{3}$, Pei-Jer Chen ${ }^{4}$, Regina Rendas-Baum ${ }^{5}$ and Dickens Theodore ${ }^{6}$

\begin{abstract}
Background: Despite changes in the treatment paradigm towards non-interferon-based therapies, interferon-based treatments are still used in some geographical regions for treating patients with hepatitis $C$ virus (HCV) infection. Use of eltrombopag with interferon-based treatment for patients with thrombocytopenia and HCV was assessed in two similarly designed phase 3 trials (Eltrombopag to Initiate and Maintain Interferon Antiviral Treatment to Benefit Subjects With Hepatitis C-Related Liver Disease [ENABLE-1 and ENABLE-2]). These trials also aimed to determine whether response to antiviral therapy (e.g., sustained virologic response [SVR]) is associated with changes in health-related quality of life (HRQoL). This pooled, post-hoc analysis aimed to (1) determine whether or not specific aspects of clinical response to treatment (i.e., achieving SVR) are associated with a significant change in HRQoL, and (2) to determine the magnitude and direction of the association between important changes in HRQoL, clinical response to interferon-based therapy (e.g., SVR) and treatment (eltrombopag or placebo), and patient and disease attributes.
\end{abstract}

Methods: The Short-Form 36 Health Survey version 2 and Chronic Liver Disease Questionnaire-Hepatitis C Virus version were administered at various time points during the studies. Results from both trials were pooled for the analyses. Logistic regression analysis was used to assess the influence of 5 clinical factors (SVR, early virologic response [EVR], genotype [2/3 vs. non-2/3], treatment [eltrombopag or placebo], and cumulative interferon dose), plus other factors including ethnicity, model of end-stage liver disease score, and platelets as predictors of meaningful changes in HRQoL.

Results: Between antiviral therapy baseline and the end of the 24-week post-treatment follow-up, declines in HRQoL were smaller in eltrombopag-treated patients than in placebo-treated patients, but the differences were not statistically significant. Mean changes among patients achieving SVR and EVR were small in comparison to thresholds of minimally important changes. Logistic models did not confirm the strength of the 5 clinical factors as predictors of meaningful changes in HRQoL during antiviral therapy, with the exception of the interaction between SVR and EVR $(P=0.0009)$. Asian ethnicity had a consistent effect on HRQoL, with East Asian patients being more likely to experience deterioration in HRQoL compared with white and/or other non-East Asian patients.

* Correspondence: kmgrotzinger@embarqmail.com

${ }^{1}$ GlaxoSmithKline, 1250 S. Collegeville Road, Collegeville, PA 19426, USA

Full list of author information is available at the end of the article 
(Continued from previous page)

Conclusions: While on active antiviral therapy, declines in HRQoL were not statistically different for eltrombopagtreated patients versus placebo-treated patients, suggesting that eltrombopag neither worsened HRQoL nor mitigated the effects of antiviral therapy on HRQoL.

Keywords: Eltrombopag, Hepatitis C virus, HRQoL, Peginterferon alfa-2a, Ribavirin, Virologic response

\section{Background}

Thrombocytopenia is a frequent complication in patients with chronic hepatitis $\mathrm{C}$ virus (HCV) infection and correlates with liver disease severity [1]. It is more severe in patients with advanced disease undergoing interferon (IFN)-based antiviral therapy (AVT) and can be attributed to antiviral infection-induced liver cirrhosis and the inhibitory effects of IFN on thrombopoietin-induced development of megakaryocytic progenitor cells [1-4].

Chronic HCV infection is associated with reduced health-related quality of life (HRQoL), having a greater impact in patients with more severe liver disease [5]. Although decreased HRQoL was documented during the course of therapy with pegylated IFN (PEG-IFN) and ribavirin, multiple studies showed that HRQoL is restored to pre-AVT levels following stopping of IFNbased AVT after achieving a sustained virologic response (SVR) [6-11].

Eltrombopag (Promacta, GlaxoSmithKline, Research Triangle Park, NC, USA) is an oral, nonpeptide thrombopoietin receptor agonist approved for the treatment of thrombocytopenia in patients with chronic HCV to allow the initiation and maintenance of IFN-based AVT based on the ENABLE-1 and ENABLE-2 trials (ClinicalTrials.gov identifiers: NCT00516321 and NCT00529568, respectively) [12]. The ENABLE trials included a difficult-to-treat group of thrombocytopenic HCV-infected patients otherwise ineligible for IFN-based AVT based on their platelet counts.

The pivotal ENABLE trials not only established the efficacy and safety of eltrombopag in patients with HCVassociated thrombocytopenia, but also assessed HRQoL through the Short-Form 36 Health Survey version 2 (SF-36v2) and Chronic Liver Disease QuestionnaireHCV version (CLDQ-HCV) [13-15]. Although effective, therapy with IFN and ribavirin has side effects such as flulike symptoms, anemia, and emotional changes $[16,17]$ that affect HRQoL [5, 18]. Based on published reports documenting the negative effects of IFN-based AVT on HRQoL $[19,20]$, HRQoL in patients in the ENABLE studies was expected to decline during the 24-48 weeks of IFN therapy due to the cumulative nature of the effects of IFN, and to return to baseline levels at the 24-weeks post-treatment follow-up visit [19]. Several reports indicate that patients who achieve SVR have improved HRQoL compared to patients who do not achieve SVR [7-11, 21, 22]. This pooled, post-hoc analysis aimed to
(1) determine whether or not specific aspects of clinical response to treatment, such as achieving a SVR, are associated with a significant change in HRQL, and (2) to determine the magnitude and direction of the association between important changes in HRQoL, clinical response to IFN-based AVT (e.g., SVR) and treatment (eltrombopag or placebo), and patient and disease attributes.

\section{Methods \\ Study design}

The similarly designed ENABLE studies (Fig. 1) differed in the PEG-IFN used with oral ribavirin (ENABLE-1, PEG-2a; ENABLE-2, PEG-2b) and the platelet thresholds for initiating AVT (ENABLE-1, $90 \times 10^{9} / \mathrm{L}$; ENABLE-2, $100 \times 10^{9} / \mathrm{L}$ ) [12]. The protocols for both studies were reviewed and approved by the applicable ethics committee or institutional review boards at each center, in accordance with the International Conference on Harmonisation guidelines, applicable country-specific requirements, and the ethical principles of the 2008 Declaration of Helsinki. Written informed consent was obtained from all participants. Each study included a 2-9-week open-label phase during which the eltrombopag dose was titrated based on platelet response (Fig. 1). Patients not achieving platelet thresholds despite receiving eltrombopag $100 \mathrm{mg} /$ day for 3 weeks entered the follow-up period. End of treatment was defined as the end of the planned AVT duration (24 weeks for genotypes $2 / 3,48$ weeks for non-genotypes $2 / 3$, or withdrawal, whichever occurred first).

The American Association for the Study of Liver Diseases guidelines for stopping and futility were applied to PEG-IFN treatment [23]. Patients were stratified by HCV genotype ( $2 / 3$ vs. non-2/3), baseline platelet counts $\left(<50 \times 10^{9} / \mathrm{L}\right.$ vs. $\left.\geq 50 \times 10^{9} / \mathrm{L}\right)$, and $\mathrm{HCV}$ viral load $(<800,000$ vs. $\geq 800,000 \mathrm{IU} / \mathrm{mL})$ at baseline [12]. All individuals involved in the study were blinded to treatment assignments.

PEG-IFN dose modifications were determined by local package instructions. The eltrombopag dose was reduced if the platelet count was $>200 \times 10^{9} / \mathrm{L}$, and treatment interrupted and the dose reduced if the platelet count exceeded $400 \times 10^{9} / \mathrm{L}$. 


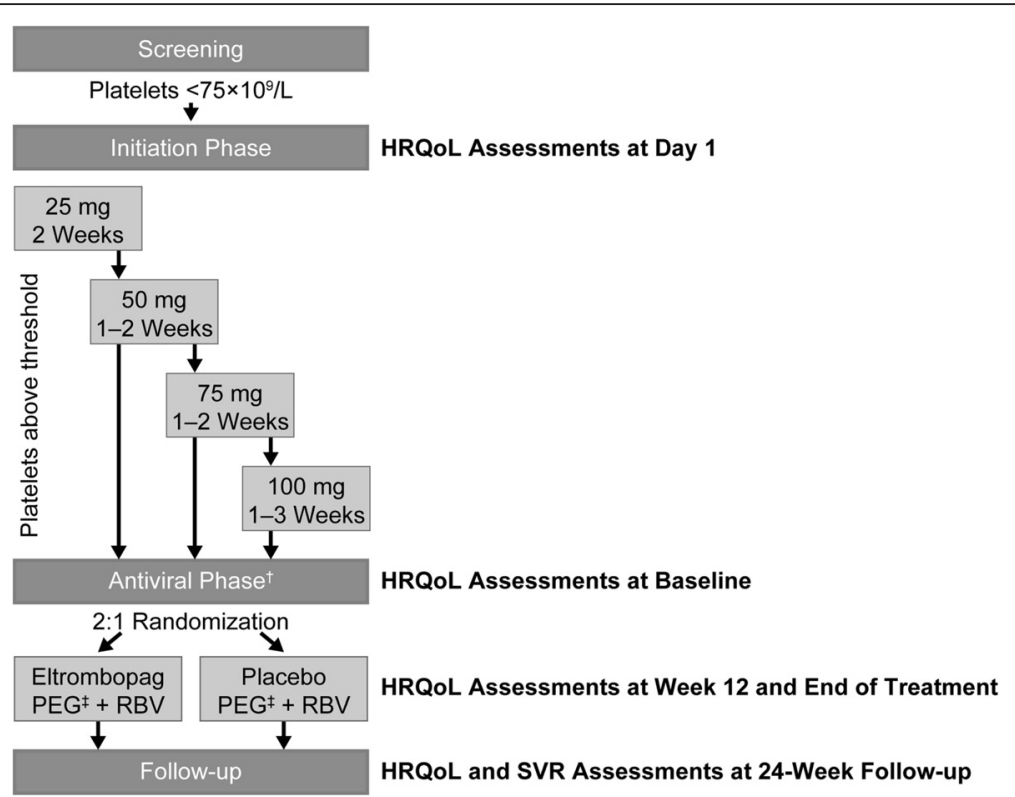

Fig. 1 Design of the ENABLE trials* and timing of the SF-36V2 and CLDQ-HCV (HRQoL) assessments. *Each study included an open-label initiation phase, with patients receiving eltrombopag 25-100 mg for up to 9 weeks, with dose escalations being guided by platelet response. Patients whose platelet counts increased to a prespecified threshold to initiate AVT (ENABLE-1, $90 \times 10^{9} / \mathrm{L} ; \mathrm{ENABLE}-2,100 \times 10^{9} / \mathrm{L}$ ) were randomized 2:1 to receive either the same dose of eltrombopag they had received during the initiation phase or matching placebo. Hepatocellular carcinoma and portal vein thrombosis were assessed between screening and baseline and every 6 months thereafter through Doppler ultrasound of the abdomen. During the AVT phase, patients in ENABLE-1 were administered PEG-2a at $180 \mathrm{\mu g} /$ week with RBV at $800 \mathrm{mg} /$ day if they were infected with genotypes $2 / 3$. If they were infected with genotypes other than 2/3, RBV doses were determined by body weight, with patients weighing $<75 \mathrm{~kg}$ being given $1000 \mathrm{mg} /$ day and patients weighing $\geq 75 \mathrm{~kg}$ being given $1200 \mathrm{mg} /$ day. The weekly PEG-2b dose in ENABLE-2 was $1.5 \mathrm{\mu g} / \mathrm{kg}$, while RBV doses were $800 \mathrm{mg} / \mathrm{day}$, $1000 \mathrm{mg} /$ day, $1200 \mathrm{mg} /$ day, or $1400 \mathrm{mg} /$ day based on body weights of $\leq 65 \mathrm{~kg}, 65-80 \mathrm{~kg}, 81-105 \mathrm{~kg}$, or $>105 \mathrm{~kg}$, respectively. ${ }^{\dagger}$ Twenty-four weeks for genotypes $2 / 3$ and 48 weeks for other genotypes of hepatitis $C$ virus. ${ }^{\ddagger}$ PEG-2a in ENABLE- 1 and PEG-2b in ENABLE-2. AVT, antiviral therapy; CLDQ-HCV, Chronic Liver Disease Questionnaire-Hepatitis C Virus version; HRQoL, health-related quality of life; PEG, pegylated interferon; RBV, ribavirin; SF-36V2, Short-Form 36 Health Survey version 2; SVR, sustained virologic response

SVR was defined as continued non-detectable $\mathrm{HCV}$ RNA 24 weeks after completion of AVT (generally 48 or 72 weeks following initiation of AVT for genotypes $2 / 3$ or at 72 weeks for non-genotype 2/3). EVR was defined as a reduction in HCV RNA $\left(\geq 2 \log _{10}\right.$ drop or undetectable RNA) after 12 weeks of antiviral treatment. HRQoL was assessed at five time points during the ENABLE trials: baseline prior to receiving eltrombopag only (Eltrombopag Alone), at the time of randomization prior to INF-based therapy, Week 12, end of IFN-based therapy, and at the 24-week follow up visit (Fig. 2). However, only three HRQoL assessments are considered in this analysis; those done at the time of randomization, at the end of IFN-based therapy, and at the 24-week follow up visit, which corresponded to the final assessment of SVR. HRQoL was assessed prior to any interaction with physicians or staff, or release of information regarding viral load.

\section{Measures of HRQoL}

This study used 2 patient-reported outcome instruments: SF-36v2 and CLDQ-HCV [6, 13].

\section{Acute recall form of the SF-36v2}

The SF-36 is a 36-item survey of self-reported functional health and well-being reported over 7 days [13]. Responses to 35 of the 36 items enable computation of a profile of functional health and well-being that consists of 8 subscales or HRQoL domains: physical functioning, role limitations due to physical health (role-physical), bodily pain, general health perceptions, vitality, social functioning, role limitations due to emotional problems (role-emotional), and mental health. Physical and mental health component summary (PCS and MCS, respectively) scores are then computed using the 8 subscales, to provide a broader metric of physical and mental HRQoL. The technique of norm-based scoring (NBS) is used to calculate the subscales and composite scores; this technique transforms or standardizes scale and component scores using the means and standard deviations (SDs) from a general US population normative sample (QualityMetric 2009 PRO Norming Study, QualityMetric, Lincoln, RI, USA; Additional file 1), drawn from a national probability sample of US non-institutionalized adults 


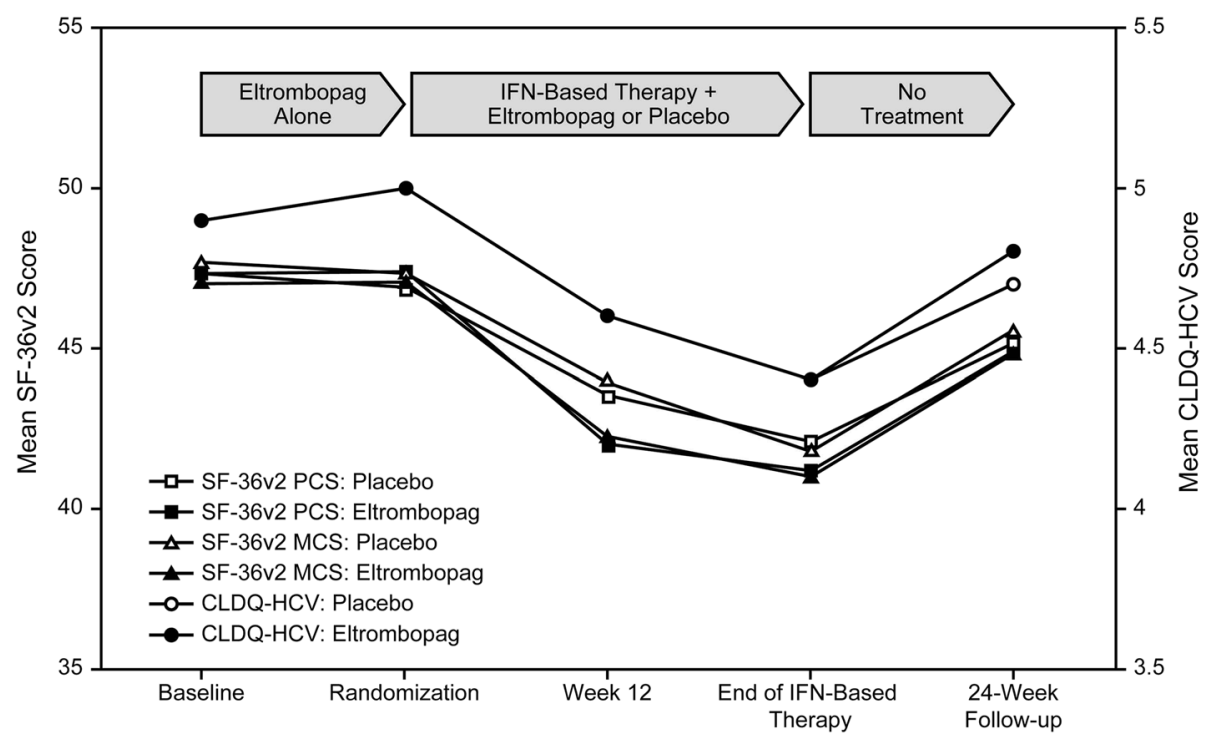

Fig. 2 Mean values over time for SF-36V2 and CLDQ-HCV. CLDQ-HCV, Chronic Liver Disease Questionnaire-Hepatitis C Virus version; IFN, interferon; MCS, mental component summary; PCS, physical component summary; SF-36v2, Short-Form 36 Health Survey version 2

(KnowledgePanel, GfK Custom Research North America, New York, NY, USA). The NBS in the general US population is set to a mean $\pm \mathrm{SD}$ of $50 \pm 10$ (i.e., T scores). Consequently, all scores $<50$ can be interpreted as being below, and scores $>50$ can be interpreted as being above, the general US population norm. All scales and component scores with NBS are scored on a common metric and facilitate meaningful comparisons. For more information on the calculation of NBS scale scores, see the Additional file $1[13,24]$.

Scores for the SF-36v2 PCS and MCS were derived according to the algorithm described by Farivar and colleagues [24] (Additional file 1). This algorithm differs from the original SF-36v2 with regard to summary score calculation in that it uses a correlated (oblique) physical and mental health factor model in lieu of the uncorrelated (orthogonal) factor solution used in the original SF-36v2.

The SF-36v2 has been used previously among patients with $\mathrm{HCV}$ and advanced fibrosis, had good measurement properties, and was responsive to change over time within patient populations without cirrhosis and with less severe liver disease $[10,25]$. The literature suggests the SF-36v2 is capable of differentiating between patients with chronic $\mathrm{HCV}$ who do and do not achieve SVR. Those achieving SVR report better HRQoL, especially on role-physical and general health domains [7-11, 21, 22]. Generally, chronic HCV infection has a pronounced impact on the vitality, general health, physical function, and social function dimensions of the SF-36 $[5,9]$.

\section{CLDQ-HCV}

The CLDQ-HCV, which is based on the CLDQ $[6,15]$, is a 29-item patient self-reported questionnaire developed to measure HRQoL among patients with chronic liver disease and HCV. Approximately one-half of the questions in the CLDQ and the CLDQ-HCV are common. The remaining questions focus on symptoms and issues unique to $\mathrm{HCV}$. The CLDQ-HCV was designed with a 2-week recall period, and items fall into 4 domains: activity/energy, emotion, systemic symptoms, and worry. Each item uses 7-point Likert scales ranging from 1 (all the time) to 7 (none of the time).

Responses on the CLDQ-HCV were coded into domain scores according to the algorithms designed by the developers; greater scores indicate better HRQoL. This permits decrements in HRQoL from baseline to be captured as negative values, while improved status is captured as positive values. Domain scores were calculated by averaging the responses within each domain, with the responses ranging from 1 to 7 . An overall score was calculated by averaging scores across the 4 domains.

\section{Statistical analysis}

Differences between groups for normally distributed variables were evaluated and tested for statistical significance using analysis of variance (ANOVA) or analysis of covariance (ANCOVA) models. When comparing means among three or more groups, if significant differences were observed from omnibus ANOVA or ANCOVA models, post- 
hoc pair-wise comparison of means were computed using Tukey's test to control for alpha inflation. The Chi-Square and Fisher's exact tests were used to assess statistical significance related to contingency tables.

Differences between groups were interpreted using Cohen's d [26]. For analyses of change scores, Cohen's d was calculated as the ratio of the difference in mean change scores between the eltrombopag and placebo groups versus the pooled standard deviation of the changes scores.

\section{Models of clinical factors associated with important changes in HRQoL}

The association between important changes in HRQoL and 5 clinical factors (SVR, EVR, genotype [2/3 vs. non$2 / 3$ ], treatment [eltrombopag vs. placebo], and cumulative dose of IFN) was evaluated using logistic regression models with the following independent variables: the 5 clinical factors, age ( $<40$ vs. $\geq 40$ years), sex, ethnicity (East Asian, other Asian, white, and other), baseline Model for End-Stage Liver Disease (MELD) score $(<10$ vs. $\geq 10)$, baseline platelet count $\left(<50 \times 10^{9} / \mathrm{L}\right.$ vs. $\geq 50 \times$ $\left.10^{9} / \mathrm{L}\right)$, and interaction terms between SVR and EVR, treatment and EVR, and genotype and cumulative IFN dose. Two dependent variables were evaluated for each HRQoL scale (8 subscales and 2 summary measures of the SF-36v2 [PCS and MCS] and 4 subscales and overall score of the CLDQ-HCV). "Drop in HRQoL score" was defined as a drop in HRQoL greater in magnitude than the minimally important change (MIC) during active AVT (end of treatment - AVT baseline). "Return to baseline score" was defined as a 24-week post-treatment follow-up HRQoL score within the MIC of the AVT baseline.

The MIC for the CLDQ-HCV was defined as one-half of the SD of the AVT baseline score [27]: overall $\mathrm{HRQoL}=0.6$; energy/activity $=4.0$; emotion $=4.8$; systemic $=3.8$; and worry $=5.4$. For SF-36v2 scores derived using NBS, the recommended MIC values were used to evaluate important changes: 3.4, 4.6, 4.3, 3.4, 6.2, 7.2, 6.2, 6.9, 4.5, and 6.2, for PCS, MCS, physical functioning, role-physical, bodily pain, general health perceptions, social functioning, role-emotional, and mental health scores, respectively [13]. Each patient in the ENABLE pooled sample was classified as experiencing a meaningful change if the difference between his or her baseline and post-baseline scores was greater in absolute value than the MIC.

The predictive powers of both SVR and EVR were modeled because both characterize response to therapy. Other considerations that underlie the model include that duration of AVT depends upon genotype [28] and that exposure for $80 \%$ of the planned time and at $80 \%$ of the planned dose of IFN is associated with the greatest likelihood of sustained viral response [29].

The model used to estimate the log odds of each HRQoL outcome is as follows:

$$
\begin{aligned}
& \log \left(\frac{\mathrm{P}[\text { HRQoL outcome }=1]}{\mathrm{P}[\text { HRQoL outcome }=0]}\right) \\
&=\alpha+\beta_{1} S V R+\beta_{2} E V R+\beta_{3} \text { Gen } 2,3+\beta_{4} \text { Eltrombopag } \\
&+\beta_{5} I F N+\beta_{6}(\text { Age }<40)+\beta_{7} \text { Male }+\beta_{8}(\text { East Asian }) \\
&+\beta_{9}(\text { Other Asian })+\beta_{10} \text { MELD }(<10)+\beta_{11}(\text { Plat }<50) \\
&+\beta_{12} \text { EVR } * S V R+\beta_{13} \text { Eltrombopag } * I F N \\
&+\beta_{14} \text { Gen } 2,3 * I F N
\end{aligned}
$$

Model assumptions were evaluated for lack of fit and evidence of multicollinearity. The assumption of linearity between continuous variables and the logit of the outcome was examined using the Hosmer-Lemeshow test [30]. Excessive collinearity or other indications of model misfit or violation of model assumptions led to modifications to the proposed model specifications.

\section{Hierarchy of significance testing for HRQoL scales}

The PCS and MCS of the SF36v2 and the overall HRQoL as derived from the CLDQ-HCV were modeled first, followed by the subscales representing challenges to physical health and functioning. The subscales or domains capturing mental and emotional health were modeled last. Table 1 shows the hierarchical order for testing of the null hypotheses.

Results are presented as odds ratios (ORs; with confidence intervals) for each HRQoL outcome. Model parameters provide the OR of the HRQoL event (drop during treatment or return to baseline level at the 24-week post-treatment follow-up assessment) for the groups represented by that factor (e.g., patients who achieved SVR vs. those who did not). For the cumulative IFN dose-a continuous variable-the interpretation of the OR refers to the effect of a 1-point difference in the cumulative IFN dose.

\section{Multiplicity adjustments and hypotheses tested}

Two families of hypotheses (Specifications A and B) were defined as follows: the odds of restoring HRQoL at the Week 24 follow-up visit (Specification A) or the odds of a drop in HRQoL greater than the MIC during active AVT (Specification B), where HRQoL is measured as scores on the SF-36v2 and CLDQ-HCV. Specification A tests whether the odds of restoring HRQoL at the 24-week post-treatment follow-up visit are significantly greater for patients with one of the 5 clinical factor characteristics (e.g., SVR) compared to patients without that characteristic (e.g., non-SVR). Specification B tests whether the odds of a drop in HRQoL greater than the MIC during active AVT are significantly greater among patients with one of 
Table 1 Hierarchical order for testing of the null hypotheses

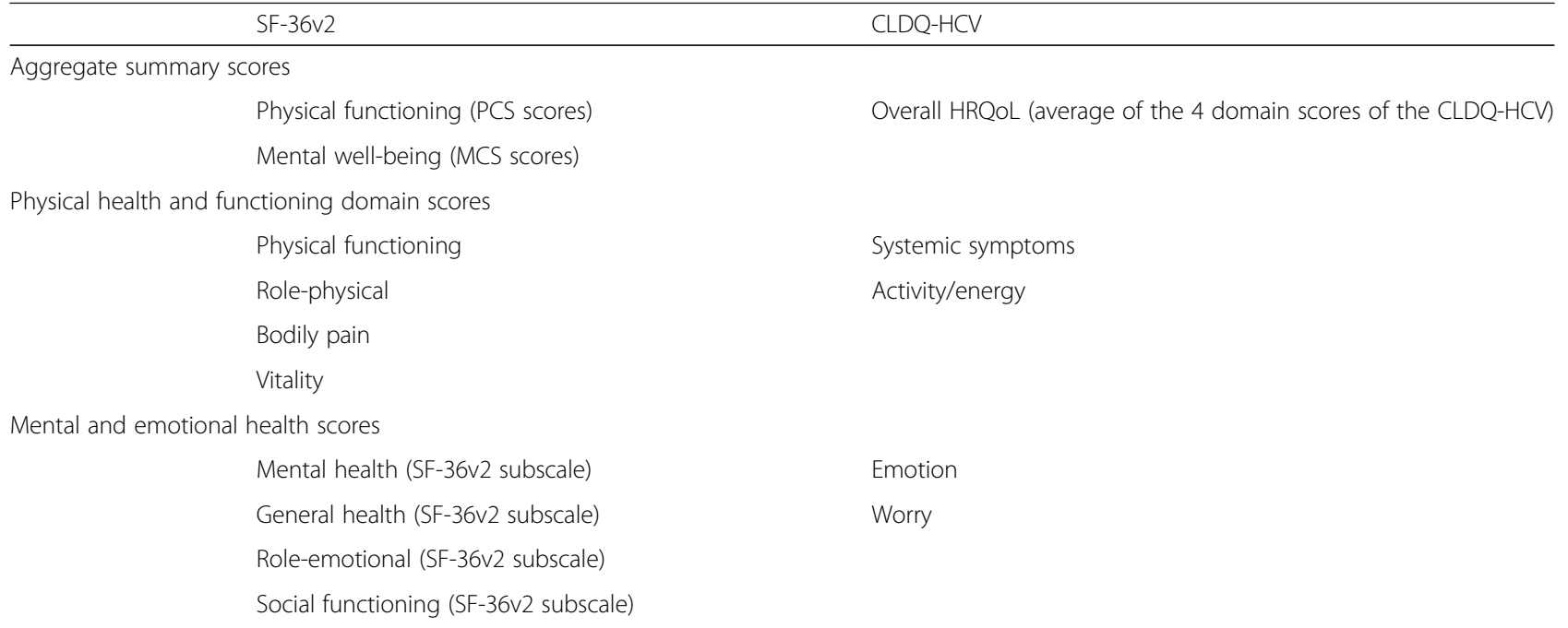

CLDQ-HCV chronic liver disease questionnaire-hepatitis C virus version, HRQoL health-related quality of life, MCS, mental component summary, $P C S$, physical component summary, SF-36v2 short-form 36 health survey version 2

the 5 clinical factor characteristics (e.g., SVR) compared to patients without that characteristic (e.g., non-SVR).

Fifteen tests were conducted for each specification, with 1 test conducted per HRQoL domain or summary score defined previously. Adjustment for multiplicity was explored. Model specifications were fitted for the purpose of answering the research questions and reflect underlying data structure assumptions, goodness-of-fit statistics, and embedded adjustments for multiple tests.

The study also examined logistic regression models (data not shown), which permitted the exclusion of particular clinical variables and/or interactions among the variables (not reported) reflecting a priori concerns of multicollinearity among clinical predictors, particularly the relationship between SVR and EVR, as well as a posteriori considerations. Type 3 analysis of effects was used to determine $P$ values for each variable included in the model using SAS/STAT(R) version 9.22 (SAS, Cary, NC, USA).

\section{Results}

Baseline demographic and clinical characteristics of the combined group of patients from the ENABLE-1 and ENABLE-2 trials $(n=1441)$ are shown in Table 2 . At baseline, mean SF-36v2 acute PCS and MCS scores (submission scoring) of the pooled patient population were 47.1 and 47.0, respectively, while respective median PCS and MCS scores were 48.6 and 48.3. The mean (median) overall CLDQ-HCV scores of the pooled population were 4.9 (5.0). Mean (median) scores on the individual components of the CLDQ-HCV were: activity, 29.7 (31.0); emotion, 44.3 (46.0); systemic symptoms, 29.1 (30.0); and worry, 38.9 (40.0).

\section{Changes in HRQoL scores across treatment and viral response groups}

Changes in mean scores for the SF-36v2 and CLDQ$\mathrm{HCV}$ over the trial period are shown in Fig. 2. The mean change in scores between Week 24 and AVT baseline ranged between -2 and -3 , indicating that SF-36v2 and CLDQ-HCV scores were lower at Week 24 than at baseline. There were small differences between the eltrombopag and placebo groups. The small group differences between Week 24 and AVT baseline, and the lack of statistical significance related to treatment in the ENABLE studies, may be partially because the number of observations was too small to make a robust estimate.

Mean changes in HRQoL scores between the end of treatment (24 or 48 weeks, depending on the genotype) and baseline are shown in Table 3 [24]. Achievement of either EVR or SVR did not explain the changes in HRQoL. There was a slight decline in SF-36v2 and CLDQ-HCV scores between AVT baseline and 24 weeks post-treatment among patients who did and did not achieve EVR (data not shown).

The association between EVR at 12 weeks post-AVT baseline and HRQoL change scores was weak, with SF$36 \mathrm{v} 2$ mean change scores that ranged from -6.5 to -3.9 among patients who did not achieve EVR and from -6.4 to -2.7 among those who achieved EVR. For the 5 CLDQ$\mathrm{HCV}$ scales, mean change scores ranged from -5.8 to -0.7 among patients who did not achieve EVR and from -5.5 to -0.5 for those who achieved EVR at 12 weeks.

Declines in SF-36v2 and CLDQ-HCV scores among those who achieved SVR (SVR group) were systematically smaller compared with patients who did not achieve SVR (non-SVR group). These group differences were 
Table 2 Baseline demographic and clinical characteristics

\begin{tabular}{|c|c|}
\hline Characteristic & $\begin{array}{l}\text { ENABLE-1 and }-2 \text { Pooled } \\
(N=1441)\end{array}$ \\
\hline \multicolumn{2}{|l|}{ Age (years) } \\
\hline Mean & 52.1 \\
\hline Median & 52.0 \\
\hline SD & 8.6 \\
\hline \multicolumn{2}{|l|}{ Baseline platelet count $\left(\times 10^{9} / \mathrm{L}\right)$} \\
\hline Mean (SD) & $56.9(13.4)$ \\
\hline Median & 59.5 \\
\hline $\begin{array}{l}\text { AVT baseline HCV RNA value transformed by } \\
\log _{10} \text {, mean (SD) }\end{array}$ & $5.7(0.8)$ \\
\hline \multicolumn{2}{|l|}{ Age, $\mathrm{n}(\%)$} \\
\hline$\geq 40$ years & $1338(92.9)$ \\
\hline$<40$ years & $103(7.2)$ \\
\hline \multicolumn{2}{|l|}{ Bridging fibrosis/cirrhosis } \\
\hline Patients with measurements, $\mathrm{n}$ & 1268 \\
\hline$n(\%)$ & $1143(90.1)$ \\
\hline \multicolumn{2}{|l|}{ Ethnicity, $n(\%)$} \\
\hline White/Other & $1109(77.0)$ \\
\hline Other Asians & $186(12.9)$ \\
\hline East Asians & $146(10.1)$ \\
\hline \multicolumn{2}{|l|}{ Actual HCV genotype, $n$ (\%) } \\
\hline Non-2/3 or missing & $995(69.0)$ \\
\hline $2 / 3$ & $446(31.0)$ \\
\hline \multicolumn{2}{|l|}{ Body mass index, $n(\%)$} \\
\hline$<30 \mathrm{~kg} / \mathrm{m}^{2}$ & $1028(71.3)$ \\
\hline$\geq 30 \mathrm{~kg} / \mathrm{m}^{2}$ & $406(28.2)$ \\
\hline Missing & $7(0.5)$ \\
\hline Depression in past medical history & $17(1.2)$ \\
\hline
\end{tabular}

$A V T$, antiviral therapy, $H C V$ hepatitis $C$ virus, $S D$ standard deviation

above the 0.2 threshold value that indicates a small group effect for all but 2 of the SF-36v2 scales: social functioning and role-emotional. The largest effect size (Cohen's $d=$ 0.56) was observed for the general health scale. For this scale, the mean change corresponded to an actual increase of 1.7 points in the SVR group and a decline of -3 points in the non-SVR group. Mean change scores experienced by both SVR and non-SVR groups were smaller than the recommended MIC of 7.2 for the general health scale. All SF$36 \mathrm{v} 2$ mean change scores were smaller than MIC estimates. Similarly, although effects for the CLDQ-HCV were $>0.3$ for all scales, the mean change scores were smaller than the MIC values in all cases.

Logistic regression analyses: clinical factors as predictors of meaningful changes in HRQoL

Results of the logistic regression analysis during AVT (from AVT baseline to end of treatment) showed no OR differences in patients' decrease in overall CLDQ-HCV score, relative to the MIC, for any of the 5 clinical factors or the specified interactions (Fig. 3a, goodness of fit is shown in Additional file 2). However, these results did show an ethnicity effect $(P=0.0025)$ : East Asians were significantly more likely to report a meaningful drop in overall CLDQ-HCV score versus non-East Asians (OR 2.09 [95 \% CI: 1.30, 3.34]), while non-East Asians were significantly less likely to report such a drop versus whites/other ethnicities (OR 0.56 [95 \% CI: 0.39, 0.80]). Although model details suggest a good fit of the model to the data, the very small $R^{2}$ value $(0.02)$ and relatively small AUC (0.56) indicate that the group of variables included in the model is a poor predictor of whether a patient's CLDQ-HCV score drops by a meaningful amount from study baseline to endpoint.

Analysis of return to AVT baseline and restoration of baseline HRQoL after completing 24 weeks of treatment are shown in Fig. 3b. None of the 5 clinical factors or viral response interaction terms was found to influence the ORs of patients' return to their AVT baseline overall CLDQ-HCV score at the 24-week post-treatment followup. These results also showed an ethnicity effect $(P=0.0207)$ : patients of East Asian ethnicity were significantly more likely to demonstrate a return to their baseline overall CLDQ-HCV score at the 24-week posttreatment follow-up versus non-East Asians (OR 1.89 [95 \% CI: 1.19, 3.02]) or whites/other ethnicities (OR 1.54 [95 \% CI: 1.07, 2.22]). Significant differences were also found regarding the MELD score $(P=0.02)$ : patients with a MELD score $<10$ were significantly more likely to demonstrate a return to their baseline overall CLDQHCV score versus those with a score $\geq 10$ at the 24-week post-treatment follow-up (OR 1.30 [95 \% CI: 1.04, 1.62]). Although the model details support a good fit to the data, the very small $R^{2}$ value (0.02) and relatively small area under the curve (AUC; 0.58 ) indicate that the group of variables included in the model is a poor predictor of whether a patient returns to his or her baseline overall CLDQ-HCV score.

Among the non-SVR group, a statistically significant decrease in the ORs of a meaningful drop in PCS scores relative to the MIC, from AVT baseline to the end of AVT, was found for patients who did not achieve EVR versus those who did (OR 0.65 [95 \% CI: 0.49, 0.85]) (Fig. 4a). No significant differences in the ORs of demonstrating a meaningful drop in PCS scores from AVT baseline to the end of AVT were found between East Asians and other ethnicities (OR 0.85 [95 \% CI: 0.59, 1.23]), although an overall race effect was demonstrated $(P=0.0064)$ due to non-East Asians being significantly less likely to experience such a drop versus whites/other ethnicities (OR 0.56 [95 \% CI: 0.39, 0.80]). 
Table 3 Drop in SF-36 and CLDQ-HCV HRQoL scores from antiviral baseline to end of ATV treatment, and at the 24-week post-treatment follow-up by treatment group

\begin{tabular}{|c|c|c|c|c|c|c|}
\hline & \multicolumn{3}{|c|}{ Drop in ATV BL to End of Treatment Mean $(95 \%$ Cl) } & \multicolumn{3}{|c|}{ Drop in ATV BL to 24-week FU Mean (95\% Cl) } \\
\hline & PBO & EPAG & Cohen's d & $\mathrm{PBO}$ & EPAG & Cohen's d \\
\hline \multicolumn{7}{|l|}{ SF-36v2 acute } \\
\hline Physical functioning & $-5.2(-6.1,-4.4)$ & $4.8(-5.4,-4.2)$ & 0.05 & $-2.2(-3.1,-1.4)$ & $-1.6(-2.2,-1.0)$ & 0.08 \\
\hline Role-physical & $-4.7(-5.7,-3.8)$ & $-5.5(-6.2,-4.8)$ & -0.08 & $2.4(-3.3,-1.4)$ & $-2.2(-2.9,-1.6)$ & 0.02 \\
\hline Bodily pain & $-4.6(-5.6,-3.7)$ & $-4.8(-5.5,-4.1)$ & -0.02 & $2.3(-3.4,-1.3)$ & $-1.7(-2.4,-1.0)$ & 0.06 \\
\hline General health & $-3.8(-4.5,-3.0)$ & $-2.9(-3.4,-2.3)$ & 0.11 & $2.3(-3.1,-1.4)$ & $-1.8(-2.4,-1.2)$ & 0.06 \\
\hline Vitality & $-6.0(-7.0,-5.1)$ & $-6.1(-6.7,-5.4)$ & -0.00 & $-2.1(-3.0,-1.2)$ & $-1.4(-2.1,-0.8)$ & 0.07 \\
\hline Social functioning & $-5.3(-6.3,-4.3)$ & $-6.5(-7.3,-5.8)$ & -0.12 & $2.5(-3.5,-1.6)$ & $-2.2(-2.9,-1.5)$ & -0.12 \\
\hline Role-emotional & $-5.3(-6.5,-4.0)$ & $-6.8(-7.7,-5.8)$ & -0.11 & $-3.1(-4.2,-2.0)$ & $-2.8(-3.7,-1.9)$ & 0.03 \\
\hline Mental health & $-5.8(-6.7,-4.9)$ & $-5.3(-6.0,-4.6)$ & 0.05 & $-2.1(-3.0,-1.1)$ & $-1.7(-2.4,-1.1)$ & 0.07 \\
\hline $\mathrm{PCS}^{\mathrm{a}}$ & $-5.8(-6.6,-5.0)$ & $-6.2(-6.8,-5.6)$ & -0.05 & $-2.8(-3.6,-1.9)$ & $-2.3(-2.8,-1.7)$ & 0.03 \\
\hline$M C S^{a}$ & $-6.3(-7.2,-5.4)$ & $-6.4(-7.1,-5.8)$ & -0.02 & $-2.6(-3.5,-1.7)$ & $-2.1(-2.7,-1.5)$ & 0.06 \\
\hline \multicolumn{7}{|l|}{ CLDQ-HCV } \\
\hline Overall & $-0.6(-0.7,-0.5)$ & $-0.6(-0.6,-0.5)$ & 0.02 & $-0.3(-0.4,-0.2)$ & $-0.2(-0.3,-0.2)$ & 0.06 \\
\hline Activity & $-5.6(-6.3,-4.8)$ & $-5.6(-6.2,-5.1)$ & -0.01 & $-1.9(-2.7,-1.2)$ & $-1.8(-2.3,-1.3)$ & 0.02 \\
\hline Emotion & $-4.8(-5.7,-3.8)$ & $-4.5(-5.1,-3.9)$ & 0.03 & $-1.9(-2.8,-1.0)$ & $-1.8(-2.4,-1.1)$ & 0.02 \\
\hline Systemic symptoms & $-4.0(-4.6,-3.3)$ & $-4.0(-4.4,-3.5)$ & -0.00 & $-2.3(-3.0,-1.6)$ & $-1.7(-2.2,-1.3)$ & 0.09 \\
\hline Worry & $-2.5(-3.4,-1.6)$ & $-1.8(-2.4,-1.2)$ & 0.07 & $-1.7(-2.7,-0.7)$ & $-0.9(-1.6,-0.2)$ & 0.08 \\
\hline
\end{tabular}

$\mathrm{Cl}$ confidence interval, CLDQ-HCV chronic liver disease questionnaire-hepatitis $\mathrm{C}$ virus version, MCS mental component summary, $P C S$ physical component summary, SF-36v2 short-form 36 health survey version 2

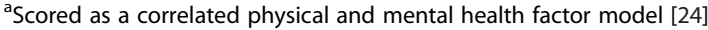

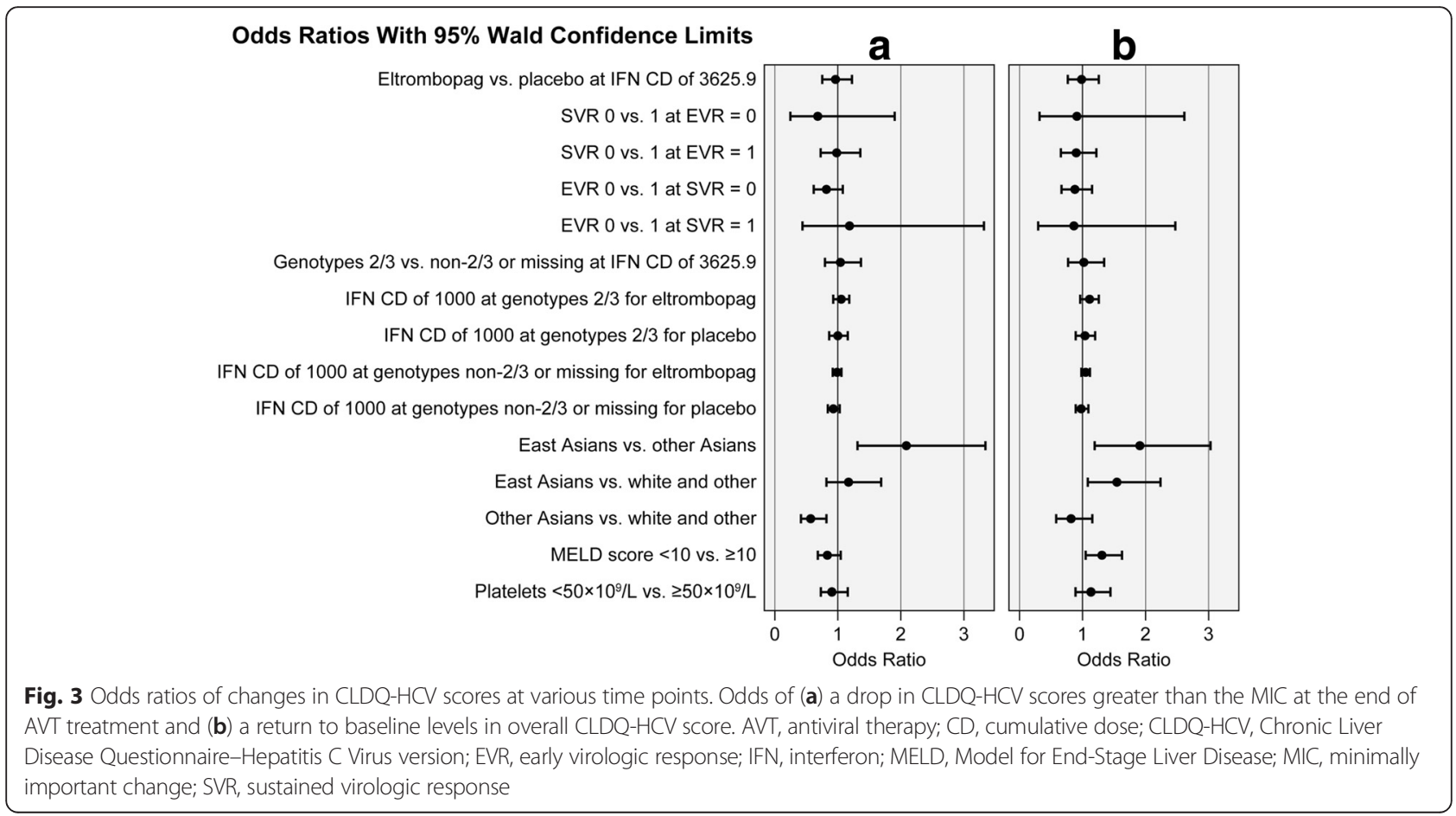




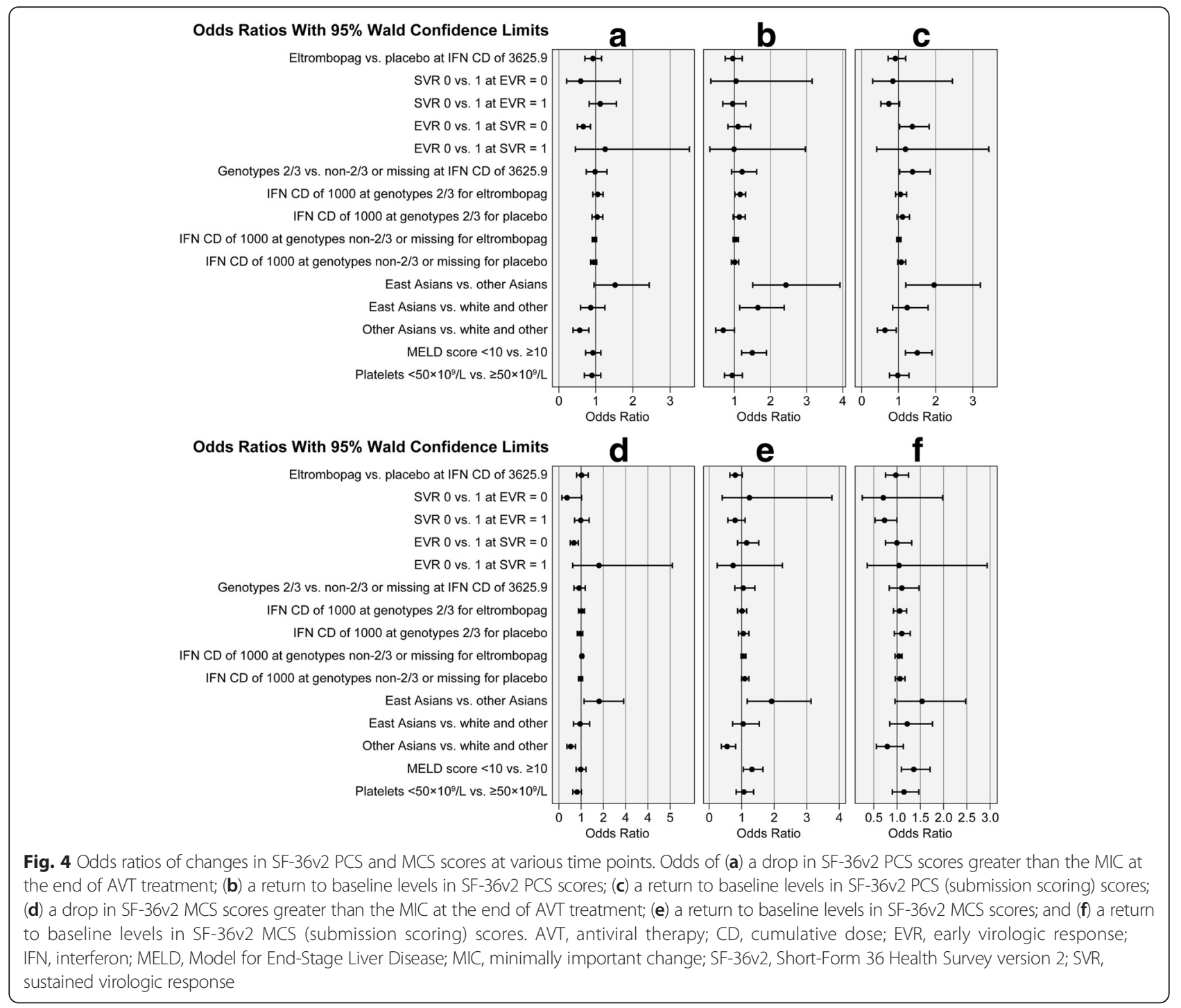

At the 24-week post-treatment follow-up visit postAVT, no differences were found in the ORs of patients' return to their baseline SF-36v2 PCS score across any of the 5 clinical factors or the specified interactions (Fig. 4b; goodness of fit is shown in Additional file 2). The results show an ethnicity effect $(P=0.0017)$ : East Asians were significantly more likely to demonstrate a return to baseline PCS score at the 24-week post-treatment follow-up versus non-East Asians (OR 2.41 [95 \% CI: 1.48, 3.92]) or whites/other ethnicities (OR 1.64 [95 \% CI: 1.13, 2.37]), while non-East Asians were significantly less likely to demonstrate a return versus whites/other ethnicities (OR 0.68 [95 \% CI: 0.46, 0.99]). Significant differences were also found regarding the MELD score $(P=0.0009)$ : patients with a MELD score $<10$ were significantly more likely to demonstrate a return to their baseline PCS score at the 24-week post-treatment follow-up versus those with a score $\geq 10$ (OR 1.48 [95\%
CI: 1.18, 1.87]). Again, model details suggest a good fit of the model to the data (Additional file 2); however, the very small $R^{2}$ value (0.02) and relatively small AUC $(0.58)$ indicate this group of variables is a poor predictor of whether a patient returns to their baseline PCS score.

Differences in the ORs of return to baseline SF-36v2 PCS (correlated physical and mental health factor model) scores at the 24-week post-treatment follow-up across some of the 5 clinical factors were also found (Fig. 4c). These differences between AVT baseline and the 24-week post-treatment follow-up included: statistically significantly greater odds for a return to baseline in PCS (correlated physical and mental health factor model) scores for patients who did not achieve EVR versus those who did (OR 1.36 [95\% CI: 1.01, 1.82]); statistically significantly larger odds of experiencing a return to AVT baseline for patients with a non-2/3 genotype (OR 1.37 [95 \% CI: 1.02, 1.84]); East Asians were 
significantly more likely to demonstrate a return to their baseline PCS (correlated physical and mental health factor model) score versus non-East Asians (OR 1.936 [95 \% CI: 1.18, 3.18]), and non-East Asians were also significantly less likely to demonstrate a return versus whites/other ethnicities (OR 0.63 [95 \% CI: 0.43, 0.92]); and a significantly increased odds of patients with a MELD score $<10$ demonstrating a return to their baseline PCS (correlated physical and mental health factor model) score (OR 1.49 [95 \% CI: 1.17, 1.89]).

Increased odds of a meaningful drop in MCS scores from AVT baseline to the end of AVT relative to the MIC were related to race $(P=0.0020)$, with East Asians more likely to experience such a drop relative to non-East Asians (OR 1.81 [95 \% CI: 1.13, 2.90]); non-East Asians were also significantly less likely to demonstrate such a drop versus whites/other ethnicities (OR 0.525 [95 \% CI: $0.37,0.75]$ ). Among the non-SVR group, patients who did not achieve EVR had a statistically significant decrease in the ORs of a drop in their MCS score that exceeded the MIC from baseline to the end of AVT versus patients who achieved EVR (OR 0.663 [95 \% CI: 0.50, 0.87]) (Fig. 4d).

No differences in the ORs of patients' return to baseline SF-36v2 MCS scores at the 24-week post-treatment follow-up were found across any of the 5 clinical factors or the specified interactions (Fig. 4e). Results show an ethnicity effect $(P=0.0072)$ : East Asians were significantly more likely to demonstrate a return to their baseline MCS score at the 24-week post-treatment follow-up visit than were non-East Asians (OR 1.91 [95 \% CI: 1.17, 3.14]), while non-East Asians were significantly less likely to demonstrate a return than were whites/other ethnicities (OR 0.550 [95 \% CI: 0.37, 0.81]). Significant differences $(P=0.0183)$ were also found regarding the MELD score: patients with a MELD score $<10$ were significantly more likely to demonstrate a return to their baseline MCS score at the 24-week post-treatment follow-up visit versus patients with a MELD score $\geq 10$ (OR 1.32 [95 \% CI: 1.05, 1.66]).

No differences in the ORs of patients' return to baseline SF-36v2 MCS (correlated physical and mental health factor model) scores at the 24-week post-treatment follow-up were found across any of the 5 clinical factors for the MCS scored using the alternate approach (Fig. 4f). However, patients with a MELD score $<10$ had significantly increased ORs of demonstrating a return to their baseline MCS (correlated physical and mental health factor model) score at the 24-week posttreatment follow-up (OR 1.36 [95 \% CI: 1.08, 1.70]).

Again, among the non-SVR group, patients who did not achieve EVR (i.e. no viral response early or late) had a statistically significant lower odds of a drop in MCS (correlated factor model) score equal or greater than the MIC between AVT initiation and end of AVT compared with patients who achieved EVR (OR 0.76 [95 \% CI: 0.58, 0.99]) (data not shown). Similarly, non-East Asians were also less likely to report a meaningful drop in MCS (correlated factor model) scores from AVT baseline to the 24-week post treatment follow-up relative to whites/ other ethnicities (OR 0.54 [95 \% CI: 0.38, 0.77]).

Results and model specifications in this article were updated from those originally submitted as part of regulatory files in order to reflect the most recent and preferred analysis algorithm from the developers of the original instruments, and further exploration and testing of goodness-of-fit criteria and underlying model econometric assumptions. Alternative models excluded specific clinical variables and/or interactions among clinical variables as described earlier, or otherwise mathematically transformed predictor variables. Tests of these alternate models did not result in fundamentally different conclusions being reached regarding the predictive variables compared with analysis using original models.

\section{Discussion}

Analyses presented in this report indicate that the physical and mental functioning of the pooled sample from the ENABLE studies were below that of the general US population, although the differences were small (on the order of $<0.5 \mathrm{SD}$ ) in most cases. SF-36 scores from previous publications $[5,9,10]$ generally agree with those in this study, particularly regarding the impact upon specific SF-36v2 HRQoL domains. All patients enrolled in the ENABLE trials had thrombocytopenia, and $90 \%$ had cirrhosis. Based on the similar safety and efficacy results of the individual ENABLE trials, the descriptive HRQoL analyses in the pooled sample is not expected to differ from HRQoL results analyzed separately.

An important finding of this study is the observed decline in HRQoL from baseline at AVT initiation and during the course of treatment, with a return to baseline levels upon completing treatment. Although declines in HRQoL were smaller in eltrombopag-treated patients, differences between this group and placebo-treated patients were generally too small to be interpreted as clinically meaningful when the change in HRQoL from baseline to the end of treatment and from AVT baseline to 24 weeks following the completion of AVT were assessed. Treatment with eltrombopag did not mitigate the effects of AVT on HRQoL or worsen adverse events or symptoms of AVT versus placebo. The data suggest that eltrombopag, by keeping platelet counts at levels sufficient to initiate, continue, and complete INF-based AVT, can provide patients who have severe liver disease an opportunity to receive IFN-based treatment without increasing adverse events such as flu-like symptoms or depression that would further impact their HRQoL. Although new non-IFN-based therapies are approved, 
these treatments may not be available to patients with $\mathrm{HCV}$ in all geographical regions of the world, where IFN may still be used to treat patients with HCV infection.

Regarding both EVR and SVR, analyses of HRQoL changes showed a greater association and clearer patterns in terms of smaller declines in mean HRQoL scores among patients achieving a virologic response. Nevertheless, mean changes in patients achieving EVR and SVR tended to be small compared with accepted thresholds of minimally (clinically) important difference. Furthermore, differences between patients achieving versus not achieving EVR were generally too small to be interpreted as meaningful. Although differences in effect sizes were slightly larger between patients who did and did not achieve SVR, these were also too small to be interpreted as meaningful. Achieving SVR and eliminating the viral infection would be anticipated to slow the progression of liver disease, but do not reverse it. Partitioning the HRQoL effects into those related to INF-based therapy versus those related to viral clearance (EVR and SVR), IFN-based treatment would likely account for the majority of the decline in HRQoL during treatment and improvement in HRQoL after stopping treatment.

Patients infected with HCV genotypes 2 and 3 received 24 weeks of AVT versus 48 weeks for patients infected with other genotypes. Nonetheless, with the exception of the small interaction between SVR and EVR, none of the 5 clinical factors (SVR, EVR, genotype [2/3 vs. non-2/3], treatment [eltrombopag or placebo], and cumulative IFN dose) was confirmed as a predictor of meaningful change in HRQoL. However, patients with a MELD score $<10$ (those with less severe cirrhosis and liver damage) were significantly more likely to return to their overall baseline CLDQ-HCV, PCS, and MCS scores.

The logistic regression models revealed consistent OR patterns for East Asians versus other Asians, and other Asians versus white/other ethnicities for the CLDQ-HCV and SF-36v2 PCS and MCS scores. East Asian patients were consistently more likely to experience a meaningful drop in HRQoL than white/other patients, but also more likely to experience a return to their baseline scores. Other Asians were generally less likely to experience a drop in HRQoL versus white/other patients. Of note, Asian patients received a reduced eltrombopag dose, although eltrombopag treatment seemed to have little impact on HRQoL changes versus placebo. Assessments of HRQoL in Asian patients with chronic HCV infections receiving interferon-based AVT have not been reported in the literature. However, cultural differences in perceptions regarding health status may contribute to differences in scores for the different scales of the SF-36v2, as was seen in a study in Taiwan that revealed the vitality scale was a stronger measure of mental health than physical health in the local population [31].
The current study has limitations. Data were derived from clinical trials, which may not fully reflect real-world clinical practice. Relatively few patients achieved SVR or EVR compared with those treated with AVT and randomized to receive eltrombopag; therefore, we may not have been able to detect robust average change in HRQoL because of the small number of responders. A general US population was used as the norm in these analyses; however, the trials were international, and differences seen in Asian patients enrolled in these studies may be affected by this. Additionally, there are many options available for measuring the MIC and no particular method is indicative of or reliable in reflecting how patients themselves may perceive change in their health status or quality of life. The choice to use one half SD as an estimate of the MIC was reasonable for expressing observed changes in a standardized way, but is also imperfect and may under- or overestimate the true MIC, as discussed by Farivar [32] in a critical review of Norman et al. [27].

\section{Conclusions}

A decline in HRQoL was observed during AVT with a return to baseline levels at 24-week post treatment followup. Treatment with eltrombopag neither significantly reduced nor enhanced the pattern of changes in HRQoL associated with AVT. Clinical factors did not appear to have large effects on HRQoL, but East Asian patients were more likely to experience declines in HRQoL during AVT.

\section{Additional files}

Additional file 1: Additional information on norm-based scoring and summary score calculations for the SF-36v2. (PDF 36 kb)

Additional file 2: Goodness of fit for logistic regression models (Figs 3 and 4). (PDF 229 kb)

\section{Abbreviations}

AIC: akaike information criterion; AUC: area under the curve; AVT: antiviral therapy; Cl: confidence interval; CLDQ-HCV: Chronic Liver Disease Questionnaire-HCV version; EVR: early virologic response; HCV: hepatitis C virus; HRQOL: health-related quality of life; IFN: interferon; Log L: log of the likelihood function; MCS: mental component summary; MELD: Model for End-Stage Liver Disease; MIC: minimally important change; NBS: norm-based scoring; OR: odds ratio; PCS: physical component summary; PEG-IFN: pegylated interferon; RBV: ribavirin; SD: standard deviation; SF-36v2: Short-Form 36 Health Survey version 2; SVR: sustained virologic response.

\section{Competing interests}

This study was supported by GlaxoSmithKline (GSK). Eltrombopag is an asset of Novartis AG as of March 2, 2015.

KM Grotzinger and D Theodore were employees at GSK during the time of study conduct and initial publication development and hold equity ownership in GSK. ZM Younossi has served as a consultant for AbbVie, Bristol-Myers Squibb (BMS), Gilead, GSK, and Intercept.

EG Giannini received travel grants and advisory arrangements from Gilead, GSK, and Janssen; provided consultancy for 4-SC and GSK; and participated in speakers' bureaus for Bayer, BMS, Gilead, GSK, Hoffman-LaRoche (Roche), Merck, and Novartis.

P-J Chen has received grants from BMS, Johnson \& Johnson, and Roche; consulting fees/honoraria from Bayer, BMS, Merck Sharp \& Dohme (MSD), 
Roche, and Taiha; travel support from BMS and Medigen; fees for participation in data monitoring boards, statistical analysis, end point committees, and the like from GSK; paid for board membership or consultancy by BMS, MSD, and Roche; and received payment from Bayer and BMS for lectures.

R Rendas-Baum received payment from GSK for conducting statistical analysis and writing the report on the statistical methods and results.

\section{Authors' contributions}

$K M G, Z M Y$, and DT contributed to the conception and design of the study. KMG, ZMY, EGG, P-JC, RR-B, and DT were involved in data analysis and interpretation. All authors contributed to the writing of the manuscript or reviewed critically and approved the final manuscript.

\section{Acknowledgments}

Editorial support in the development of the manuscript was provided by Prasad Kulkarni, PhD, and Nancy Price, PhD, of AOI Communications and funded by GlaxoSmithKline and Novartis Pharmaceuticals, Inc. KMG and DT were employees of GSK during the study.

\section{Author details}

${ }^{1}$ GlaxoSmithKline, 1250 S. Collegeville Road, Collegeville, PA 19426, USA. ${ }^{2}$ Inova Fairfax Hospital, 3300 Gallows Road, Falls Church, VA 22042, USA. ${ }^{3}$ University of Genoa, Viale Benedetto XV, no.6, Genoa 16132, Italy. ${ }^{4}$ National Taiwan University Hospital, No.1, Changde Street, Zhongzheng District, Taipei 10048, Taiwan. ${ }^{5}$ QualityMetric, 24 Albion Road, Lincoln, RI 02865, USA. ${ }^{6}$ Novartis Pharmaceuticals Corporation, 1 Health Plaza, East Hanover, NJ 07936, USA.

\section{Received: 29 June 2015 Accepted: 9 March 2016}

Published online: 22 March 2016

\section{References}

1. Giannini EG. Review article: thrombocytopenia in chronic liver disease and pharmacologic treatment options. Aliment Pharmacol Ther. 2006;23:1055-65.

2. Schmid M, Kreil A, Jessner W, Homoncik M, Datz C, Gangl A, Ferenci P, Peck-Radosavljevic M. Suppression of haematopoiesis during therapy of chronic hepatitis $C$ with different interferon alpha mono and combination therapy regimens. Gut. 2005;54:1014-20.

3. Ganser A, Carlo-Stella C, Greher J, Volkers B, Hoelzer D. Effect of recombinant interferons alpha and gamma on human bone marrow-derived megakaryocytic progenitor cells. Blood. 1987;70:1173-9.

4. Wang Q, Miyakawa Y, Fox N, Kaushansky K. Interferon-alpha directly represses megakaryopoiesis by inhibiting thrombopoietin-induced signaling through induction of SOCS-1. Blood. 2000;96:2093-9.

5. Bjornsson E, Verbaan H, Oksanen A, Fryden A, Johansson J, Friberg S, Dalgard $\mathrm{O}$, Kalaitzakis $\mathrm{E}$. Health-related quality of life in patients with different stages of liver disease induced by hepatitis C. Scand J Gastroenterol. 2009;44:878-87.

6. Dan AA, Martin LM, Crone C, Ong JP, Farmer DW, Wise T, Robbins SC, Younossi ZM. Depression, anemia and health-related quality of life in chronic hepatitis C. J Hepatol. 2006:44:491-8.

7. Ware Jr JE, Bayliss MS, Mannocchia M, Davis GL. Health-related quality of life in chronic hepatitis $C$ : impact of disease and treatment response. The interventional therapy group. Hepatology. 1999;30:550-5.

8. McHutchison JG, Ware Jr JE, Bayliss MS, Pianko S, Albrecht JK, Cort S, Yang I, Neary MP. Hepatitis interventional therapy G: The effects of interferon alpha-2b in combination with ribavirin on health related quality of life and work productivity. J Hepatol. 2001;34:140-7.

9. Bonkovsky HL, Woolley JM. Reduction of health-related quality of life in chronic hepatitis $C$ and improvement with interferon therapy. Hepatology. 1999:29:264-70. 3.

10. Bonkovsky HL, Snow KK, Malet PF, Back-Madruga C, Fontana RJ, Sterling RK, Kulig CC, Di Bisceglie AM, Morgan TR, Dienstag JL, et al. Health-related quality of life in patients with chronic hepatitis C and advanced fibrosis. J Hepatol. 2007:46:420-31.

11. Neary MP, Cort S, Bayliss MS, Ware Jr JE. Sustained virologic response is associated with improved health-related quality of life in relapsed chronic hepatitis C patients. Semin Liver Dis. 1999;19 Suppl 1:77-85.

12. Afdhal NH, Dusheiko GM, Giannini EG, Chen PJ, Han KH, Mohsin A, Rodriguez-Torres M, Rugina S, Bakulin I, Lawitz E, et al. Eltrombopag increases platelet numbers in thrombocytopenic patients with HCV infection and cirrhosis, allowing for effective antiviral therapy. Gastroenterology. 2014;146:442-52. e441.
13. Maruish ME. User's manual for the SF-36v2 Health Survey. 3rd ed. Lincoln, Rl: Quality Metric; 2011.

14. Younossi ZM, Guyatt G, Kiwi M, Boparai N, King D. Development of a disease specific questionnaire to measure health related quality of life in patients with chronic liver disease. Gut. 1999;45:295-300.

15. Two R, Verjee-Lorenz A, Clayson D, Dalal M, Grotzinger K, Younossi ZM. A methodology for successfully producing global translations of patient reported outcome measures for use in multiple countries. Value Health. 2010;13:128-31.

16. Benini F, Distefano L, Baisini O, Pigozzi MG, Lanzini A. Efficacy and tolerability of combination therapy with interferon-alfa plus ribavirin in patients with chronic hepatitis C virus infection: a single-center study in relapsers and nonresponders to previous treatment with high-dose interferon-alfa monotherapy. Curr Ther Res Clin Exp. 2003;64:140-50.

17. Witthoft T, Moller B, Wiedmann KH, Mauss S, Link R, Lohmeyer J, Lafrenz M, Gelbmann CM, Huppe D, Niederau C, Alshuth U. Safety, tolerability and efficacy of peginterferon alpha-2a and ribavirin in chronic hepatitis $C$ in clinical practice: the German open safety trial. J Viral Hepat. 2007;14:788-96. 4.

18. Chang $\mathrm{CH}$, Chen KY, Lai MY, Chan KA. Meta-analysis: ribavirin-induced haemolytic anaemia in patients with chronic hepatitis C. Aliment Pharmacol Ther. 2002;16:1623-32.

19. Conversano C, Carmassi C, Carlini M, Casu G, Gremigni P, Dell'Osso L. Interferon alpha therapy in patients with chronic hepatitis $\mathrm{C}$ infection: quality of life and depression. Hematol Rep. 2015;7:5632.

20. Lin FC, Young HA. Interferons: success in anti-viral immunotherapy. Cytokine Growth Factor Rev. 2014;25:369-76.

21. Arora S, O'Brien C, Zeuzem S, Shiffman ML, Diago M, Tran A, Pockros PJ, Reindollar RW, Gane E, Patel K, et al. Treatment of chronic hepatitis C patients with persistently normal alanine aminotransferase levels with the combination of peginterferon alpha-2a (40 kDa) plus ribavirin: impact on health-related quality of life. J Gastroenterol Hepatol.

2006;21:406-12

22. John-Baptiste AA, Tomlinson G, Hsu PC, Krajden M, Heathcote EJ, Laporte A, Yoshida EM, Anderson FH, Krahn MD. Sustained responders have better quality of life and productivity compared with treatment failures long after antiviral therapy for hepatitis C. Am J Gastroenterol. 2009;104:2439-48.

23. Ghany MG, Strader DB, Thomas DL, Seeff LB. American association for the study of liver D: diagnosis, management, and treatment of hepatitis C: an update. Hepatology. 2009;49:1335-74.

24. Farivar SS, Cunningham WE, Hays RD. Correlated physical and mental health summary scores for the SF-36 and SF-12 Health Survey, V.I. Health Qual Life Outcomes. 2007;5:54.

25. Spiegel BM, Younossi ZM, Hays RD, Revicki D, Robbins S, Kanwal F. Impact of hepatitis $C$ on health related quality of life: a systematic review and quantitative assessment. Hepatology. 2005;41:790-800.

26. Cohen J. Statistical power analysis for the behavioral sciences. 2nd ed. New York, NY: Taylor \& Francis; 1988

27. Norman GR, Sloan JA, Wyrwich KW. Interpretation of changes in health-related quality of life: the remarkable universality of half a standard deviation. Med Care. 2003;41:582-92. 5.

28. European Association for the Study of the Liver. EASL international consensus conference on hepatitis C. Paris, 26-28, February 1999, consensus statement. J Hepatol. 1999;30:956-61.

29. McHutchison JG, Manns M, Patel K, Poynard T, Lindsay KL, Trepo C, Dienstag J, Lee WM, Mak C, Garaud JJ, et al. Adherence to combination therapy enhances sustained response in genotype-1-infected patients with chronic hepatitis $C$. Gastroenterology. 2002;123:1061-9.

30. Hosmer S, Lemeshow S. Applied logistic regression. New York: Wiley and Sons; 1989.

31. Tseng HM, Lu JF, Gandek B. Cultural issues in using the SF-36 health survey in Asia: results from Taiwan. Health Qual Life Outcomes. 2003;1:72.

32. Farivar SS, Liu H, Hays RD. Half standard deviation estimate of the minimally important difference in HRQOL scores? Expert Rev Pharmacoecon Outcomes Res. 2004:4:515-23. 